\title{
Surface Hydrophobicity of Spores of Bacillus spp.
}

\author{
By TOMIHIKO KOSHIKA WA, * MAYUMI YAMAZAKI, \\ MASAYOSHI YOSHIMI, SETSUKO OGAWA, ATSUMI YAMADA, \\ KAZUHITO WATABE AND MITSUO TORII \\ Department of Environmental Health Sciences, Faculty of Pharmaceutical Sciences, \\ Setsunan University, Hirakata, Osaka, 573-01, Japan
}

(Received 7 March 1989; revised 5 June 1989; accepted 11 July 1989)

The surface hydrophobicity of 12 strains of Bacillus spp. was examined in a hexadecaneaqueous partition system. Mature and germinated spores of Bacillus megaterium QM B1551 transferred to the hexadecane layer, while vegetative and sporulating cells did not. Wild-type spores were more hydrophobic than spores of an exosporium-deficient mutant of B. megaterium QM B1551, although the mutant spores were shown to be hydrophobic to some extent by using increased volumes of hexadecane. This result suggests that the exosporium is more hydrophobic than the spore coat and that the surface hydrophobicity of spores depends mainly on components of the exosporium. The surface hydrophobicity of spores of nine other species of Bacillus was also examined, and spores having an exosporium were more hydrophobic than those lacking an exosporium. Thus measurement of the hydrophobicity of spores by the hexadecane partition method may provide a simple and rapid preliminary means of determining the presence or absence of an exosporium.

\section{INTRODUCTION}

The surface hydrophobicity of bacterial cells has been determined by several methods, based on the precipitation of cells by salts (Lindahl et al., 1981), hydrophobic interaction chromatography (Smyth et al., 1978; Doyle et al., 1984), and adherence to various liquid hydrocarbons including hexadecane (Rosenberg et al., 1980; Doyle et al., 1984; Craven \& Blankenship, 1987; Kutima \& Foegeding, 1987). The use of a hexadecane-aqueous partition system is one of the simplest and most rapid methods.

Spores of Bacillus cereus (Doyle et al., 1984; Kutima \& Foegeding, 1987), Clostridium perfringens (Craven \& Blankenship, 1987) and Bacillus subtilis (Doyle et al., 1984) have hydrophobic characteristics, but their affinities for liquid hydrocarbons are different. Rosenberg et al. (1985) reported that gramicidin $\mathrm{S}$ was responsible for the hydrophobicity of $B$. brevis spores, but the structural components involved are still unknown. The factors responsible for the hydrophobicity of spores of other species of Bacillus have not been reported. In morphological classification, spores may be divided into two groups based on the presence of an exosporium. Since the chemical composition of the exosporium (Matz et al., 1970; Takumi et $a l ., 1979$ ) is different from that of the spore coat (Warth et al., 1963; Aronson \& Pandey, 1978), the hydrophobic properties of spores with and without exosporium may be different. If so, it could be possible to determine the presence or absence of an exosporium on the basis of differences in surface hydrophobicity of spores.

In the present study we used a hexadecane-aqueous partition system to examine the hydrophobic characteristics of Bacillus megaterium QM B1551 at different stages of its life cycle, and those of spores of nine other species of Bacillus. We also examined the link between hydrophobicity and the presence of an exosporium. 
Table 1. Cultivation conditions and concentrations of Urografin used for purification of spores

Organism* $\quad$ Medium $\dagger \quad \begin{array}{cc}\text { Incubation } & \text { Incubation } \\ \text { time (d) } & \text { temp. }\left({ }^{\circ} \mathrm{C}\right)\end{array}$
B. brevis IFO 3331
B. cereus $\mathrm{T}$
B. circulans IFO 3329
B. coagulans IFO 3557
B. licheniformis IFO 12195
B. macerans IFO 3490
B. megaterium QM B1551
B. megaterium ATCC 33729
B. megaterium ATCC 19213
B. pumilus IFO 3813
B. subtilis ATCC 6051
$B$. thuringiensis IFO 13865
TYEA
TYEA
TYEA
BHIA
TYEA
TYEA
SNB
TYEA
SNB
TYEA
TYEA
TYEA
TYEA
TYEA

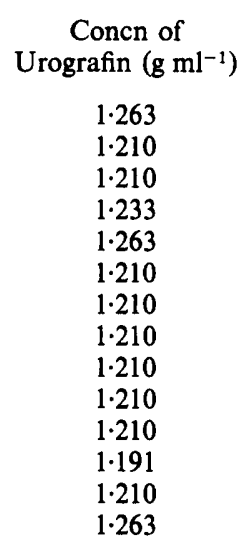

* IFO, Institute for Fermentation, Osaka; ATCC, American Type Culture Collection.

† SNB, supplemented nutrient broth; TYEA, tryptose yeast extract agar medium; BHIA, brain heart infusion agar medium.

\section{METHODS}

Organisms and media. The Bacillus strains used in this study, and their cultivation media, are listed in Table 1. Brain heart infusion agar (BHIA) contained $\left(\mathrm{g}^{-1}\right)$ : brain heart infusion (Difco), 37; yeast extract (Difco), 5 ; and agar, 15 ; in salt solution ( $\mathrm{pH} \mathrm{7.0)}$. Tryptose yeast extract agar (TYEA) contained ( $\mathrm{g} \mathrm{l}^{-1}$ ): tryptose (Difco), 10; yeast

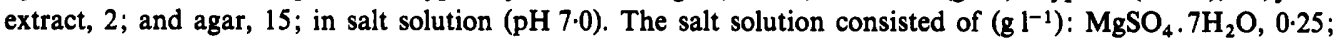
$\mathrm{MnCl}_{2} .4 \mathrm{H}_{2} \mathrm{O}, 0.025 ; \mathrm{CaCl}_{2} .2 \mathrm{H}_{2} \mathrm{O}, 0 \cdot 15$; and $\mathrm{FeSO}_{4} .7 \mathrm{H}_{2} \mathrm{O}, 0.0003$. Supplemented nutrient broth (SNB) was prepared as described by Setlow \& Kornberg (1969).

Preparation of spores. The cultivation conditions are given in Table 1. Spores were harvested and collected by centrifugation at $1500 \mathrm{~g}$ for $15 \mathrm{~min}$. The pellets were suspended in deionized water, layered on top of a solution of Urografin (Schering; the concentrations of Urografin used are indicated in Table 1) and centrifuged at $1500 \mathrm{~g}$ for $30 \mathrm{~min}$. The pellets were resuspended in deionized water, and the suspension was layered on top of a fresh Urografin solution and centrifuged. This procedure was repeated several times to remove vegetative cells and cell debris. The clean spores thus obtained were washed five times with chilled deionized water by centrifugation at $1500 \mathrm{~g}$ for $15 \mathrm{~min}$.

Preparation of vegetative cells, sporulating cells and germinated spores of B. megaterium QM B1551. Cells were inoculated into $100 \mathrm{ml}$ supplemented nutrient broth and incubated at $30^{\circ} \mathrm{C}$ with aeration by shaking for $7 \mathrm{~h}$ (vegetative cells) and for $16 \mathrm{~h}$ (sporulating cells). The cells were then harvested and washed several times with saline by centrifugation at $1500 \mathrm{~g}$ for $15 \mathrm{~min}$. After $16 \mathrm{~h}$ cultivation, more than $95 \%$ of forespores within the mother cells were refractile when observed by phase-contrast microscopy. The spore suspensions were heatactivated at $60^{\circ} \mathrm{C}$ for $30 \mathrm{~min}$. Germinated spores (more than $90 \%$ of spores having lost refractility) were prepared by incubating heat-activated spores with $10 \mathrm{mM}-\mathrm{KNO}_{3}$ at $37^{\circ} \mathrm{C}$ for $1 \mathrm{~h}$.

Measurement of the surface hydrophobicity of spores and cells. The surface hydrophobicity of mature spores was measured by the method of Rosenberg et al. (1980) with a minor modification. Spores were suspended in deionized water to prevent ionic germination. Various volumes of hexadecane (up to $1.0 \mathrm{ml}$ ) were added to $3 \mathrm{ml}$ of spore suspensions $\left(\mathrm{OD}_{610} 0.5\right)$ in test tubes $(18 \times 180 \mathrm{~mm})$, and the mixtures were agitated vigorously by Vortex mixer for $1 \mathrm{~min}$ at room temperature. After the two layers had separated completely on standing for about $15 \mathrm{~min}$, the $\mathrm{OD}_{610}$ of the aqueous phase was measured with a Shimazu UV-240 spectrophotometer. Results are presented as percentage transfer to the hexadecane layer, calculated by the formula $\left[\left(D_{\mathrm{i}}-D_{\mathrm{f}}\right) / D_{\mathrm{i}}\right] \times 100$, where $D_{\mathrm{i}}$ is the optical density of the initial spore suspension and $D_{f}$ is that of the aqueous phase after agitation with hexadecane. Hydrophobicity measurements were carried out in duplicate. The mean values from three experiments are reported; the calculated values from three experiments agreed within $\pm 3 \%$.

Hydrophobicities of vegetative cells, sporulating cells and germinated spores were determined using saline instead of deionized water as a suspension medium. 


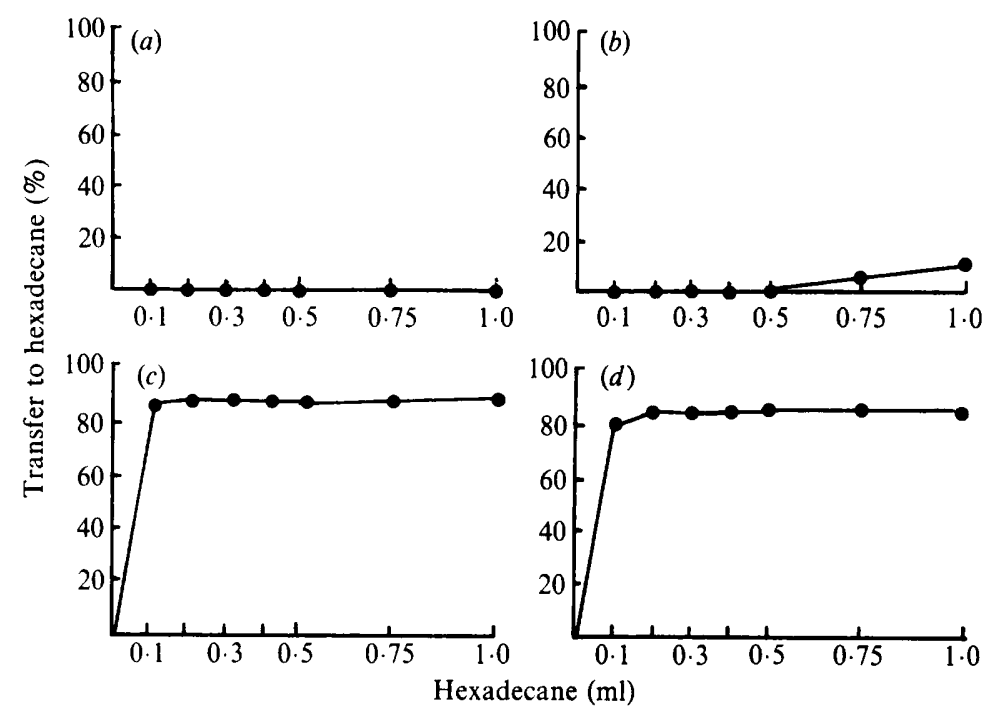

Fig. 1. Surface hydrophobic properties of $(a)$ vegetative cells, $(b)$ sporulating cells, $(c)$ mature spores and $(d)$ germinated spores of $B$. megaterium QM B1551. The cell or spore suspension ( $3 \mathrm{ml})$, prepared as described in Methods, was mixed with hexadecane (from 0 to $1.0 \mathrm{ml}$ ). The percentage transfer to hexadecane was determined by measuring the $\mathrm{OD}_{610}$ of the aqueous phase (see Methods). Each point represents the mean of three experiments (the same applies in Figs 2 and 3).

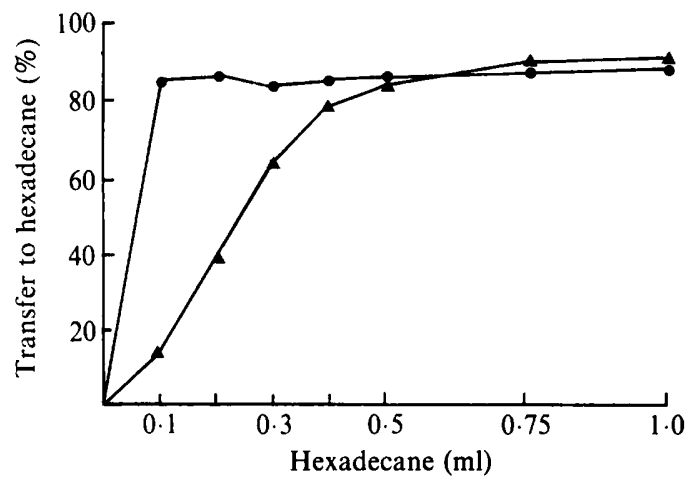

Fig. 2. Comparison of surface hydrophobic properties of wild-type ( $\bullet$ ) and exosporium-deficient $(\Delta)$ spores of B. megaterium QM B1551. Spores were prepared as described in Methods. Percentage transfer to hexadecane was determined as for Fig. 1.

\section{RESULTS}

Surface hydrophobicity of spores and cells of $B$. megaterium $Q M B 1551$

The surface hydrophobic properties of various types of $B$. megaterium QM B1551 cells are shown in Fig. 1. No vegetative cells transferred to the hexadecane layer. Sporulating cells did not transfer to hexadecane when the volume of hexadecane was $0.5 \mathrm{ml}$ or less, but minor transfer (less than $10 \%$ ) was observed with 0.75 and $1.0 \mathrm{ml}$ hexadecane. In contrast, more than $80 \%$ of the mature and germinated spores transferred to $0.1 \mathrm{ml}$ hexadecane in this partition system, which indicates that spores, either mature or germinated, are considerably hydrophobic.

Comparison of the hydrophobicity of wild-type and exosporium-deficient mutant spores of $B$. megaterium $Q M B 1551$

The spore structure of $B$. megaterium QM B1551 consists of the core, inner membrane, cortex, 


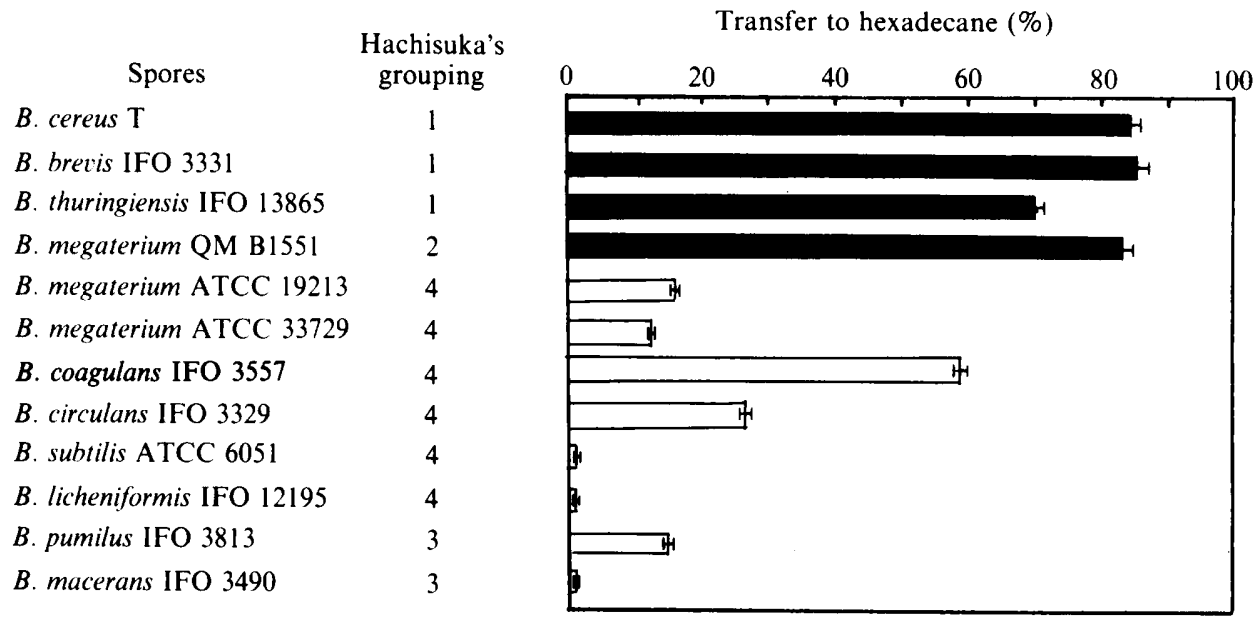

Fig. 3. Relationship between surface hydrophobicity and presence of exosporium. Spores in deionized water $(3 \mathrm{ml})$ were mixed with $0 \cdot 1 \mathrm{ml}$ hexadecane. Percentage transfer to hexadecane was determined as for Fig. 1. $\square$, Presence of exosporium based on more than $70 \%$ transfer; $\square$, absence of exosporium based on less than $70 \%$ transfer; the error bars represent SE. The classification of spores according to Hachisuka et al. (1984) is shown. The groups are defined as follows: group 1, exosporium and appendages present; group 2, exosporium only; group 3, appendages only; group 4, neither exosporium nor appendages present.

outer membrane, spore coat and exosporium (Koshikawa et al., 1984). In order to study the role of the exosporium in hydrophobicity, the surface hydrophobicity of exosporium-deficient mutant spores (Koshikawa et al., 1984) of B. megaterium QM B1551 was compared with that of wild-type spores (Fig. 2). The wild-type spores transferred easily to the hexadecane layer with only $0.1 \mathrm{ml}$ of hexadecane, while little transfer of the exosporium-deficient spores occurred with this volume of hexadecane. Transfer of the exosporium-deficient spores into hexadecane gradually increased with increasing volumes of hexadecane, but transfer was less than $80 \%$ below $0.4 \mathrm{ml}$ hexadecane. Transfer of the exosporium-deficient spores reached the same level as that obtained by the wild-type spores with $0.5 \mathrm{ml}$ hexadecane or more. These results indicate that the hydrophobic property of spores is mainly due to the exosporium, while the spore coat has a (weaker) hydrophobic property.

\section{Comparison of surface hydrophobicity of spores of various Bacillus species}

Transfer to hexadecane was determined for spores of three strains of $B$. megaterium and nine other species of Bacillus. According to their surface hydrophobic characteristics, these spores were divided into three types. The spores of B. megaterium QM B1551, B. coagulans IFO 3557, $B$. thuringiensis IFO $13865, B$. cereus $\mathrm{T}$ and $B$. brevis IFO 3331 were the most hydrophobic: more than $60 \%$ transfer occurred with $0.1 \mathrm{ml}$ hexadecane. Spores of $B$. megaterium ATCC 33729 and ATCC 19213, B. pumilus IFO 3813 and B. circulans IFO 3329 had moderate hydrophobicities, requiring $0.3-0.5 \mathrm{ml}$ hexadecane for $60 \%$ transfer. The transfer of spores of $B$. macerans IFO $3490, B$. licheniformis IFO 12195 and B. subtilis ATCC 6051 was less than $20 \%$ even with $1.0 \mathrm{ml}$ hexadecane, indicating that these bacterial spores were the least hydrophobic.

The composition of medium and the cultivation temperature affect structures and properties of spores (Hitchins et al., 1972; Waites et al., 1979; Bayliss et al., 1981; Foegeding \& Fulp, 1988). To examine the effect of culture medium on surface hydrophobicity, B. megaterium QM B1551 and ATCC 33729, B. licheniformis, B. pumilus and B. cereus $\mathrm{T}$ were cultivated in supplemented nutrient broth and on tryptose yeast extract agar and the resulting spores were tested for their hydrophobicity. The hydrophobic properties of these bacterial spores were not affected by the cultural media (data not shown).

We also examined the relationship between the presence of an exosporium and the surface 
hydrophobicity of the spores (Fig. 3). With spores possessing an exosporium (B. cereus, B. brevis, $B$. thuringiensis and B. megaterium QM B1551), transfer was more than $70 \%$, whereas for strains lacking an exosporium (except B. coagulans) transfer was less than $30 \%$.

\section{DISCUSSION}

We have studied the surface hydrophobic characteristics of spore-forming bacilli and also examined whether the measurement of surface hydrophobicity of spores could be used as indicator of the presence of an exosporium.

Mature and germinated spores of $B$. megaterium QM B1551 were strongly hydrophobic (Fig. 1). Experiments with an exosporium-deficient mutant of B. megaterium QM B1551 (Fig. 2) indicated that the hydrophobic property of spores is largely due to the exosporium, but that the spore coat makes a small contribution to hydrophobicity. In the case of the spores of $B$. brevis, gramicidin $\mathrm{S}$ was reported to be responsible for the high surface hydrophobicity (Rosenberg $e t$ $a l ., 1985)$. However, the relation of gramicidin $S$ polypeptide to the exosporium has not been established, and it is not known whether such polypeptides are produced by $\boldsymbol{B}$. megaterium.

There is little information on the chemical composition of isolated exosporia. Isolated exosporium fractions from C. botulinum type A (Takumi et al., 1979) and B. cereus (Matz et al., 1970) were composed mainly of protein and lipid. The fact that, in our experiments, spores possessing an exosporium exhibited strong hydrophobicity may be due to the protein (probably hydrophobic) and the lipid components of the exosporium.

The spore coat consists mainly of hydrophobic proteins (Warth et al., 1963; Aronson \& Pandey, 1978). Doyle et al. (1984) found that agents which disrupt protein structure modified the hydrophobicity of spores, suggesting that the spore coat also has hydrophobic sites.

Hachisuka et al. (1984) divided spores of Bacillus species into four groups on the basis of the presence of the exosporium and the appendages (see legend to Fig. 3). In our experiments, spores of $B$. brevis, B. cereus and B. thuringiensis (members of Hachisuka's group 1, with both exosporium and appendages) showed the most hydrophobic properties. The strong hydrophobic properties of these spores are believed to derive mainly from the exosporium. The contribution of appendages to their hydrophobicity is not clear at present. The reported chemical properties of the main component of isolated appendages from B. cereus IAM 1110 (Kozuka \& Tochikubo, 1985 ) would suggest that appendages may not have strong hydrophobic properties in comparison with the exosporium.

The spores of B. megaterium QM B1551 (Hachisuka's group 2, with exosporium only) showed almost the same hydrophobicity as those in group 1, which suggests that the strong hydrophobicity originates from the exosporium. Doyle et al. (1984) found that spores of $B$. anthracis (also in group 2) showed strong hydrophobicity in a hydrophobic chromatography experiment, a result which supports our conclusion.

Spores of B. macerans and B. pumilus (Hachisuka's group 3, with appendages only) had low and moderate surface hydrophobicities, respectively. The differences in surface hydrophobicities of these spores may be due to the amount of appendages or hydrophobic components in the spore coat.

Spores of B. megaterium ATCC 33729 and ATCC 19213, B. circulans, B. licheniformis, B. subtilis ATCC 6051 and $B$. coagulans belong to Hachisuka's group 4, and lack both exosporia and appendages. Their surface hydrophobicities, except for $B$. coagulans, were moderate or low. Although spores of $B$. coagulans had almost the same hydrophobicity as spores with an exosporium, the exosporium was apparently absent (Ohey \& Murrell, 1962). We do not know why $B$. coagulans spores showed strong hydrophobicity. A possible explanation is that the bacterium may have a tightly fitting exosporium-like layer as reported for $B$. subtilis PM9 (Sousa et al., 1976). The variation in hydrophobicity of spores in group 4 may reflect the relative proportion of nonpolar and polar groups on the surface of the spore coat.

Since spores possessing an exosporium showed high affinities for hexadecane, measurement of hydrophobicity with hexadecane seems to be a simple and rapid method for detecting the presence of an exosporium in Bacillus species. 


\section{REFERENCES}

Aronson, A. I. \& Pandey, N. K. (1978). Comparative structural and functional aspects of spore coats. In Spores VII, pp. 54-61. Edited by G. Chambliss \& J. C. Vary. Washington, DC: American Society for Microbiology.

Bayliss, C. E., Waites, W. M. \& KING, N. R. (1981). Resistance and structure of spores of Bacillus subtilis. Journal of Applied Bacteriology 50, 379-390.

Craven, S. E. \& Blankenship, L. C. (1987). Changes in the hydrophobic characteristics of Clostridium perfringens spores and spore coats by heat. Canadian Journal of Microbiology 33, 773-776.

Doyle, R. J., Nedjat-HaIEM, F. \& Singh, J. S. (1984). Hydrophobic characteristics of Bacillus spores. Current Microbiology 10, 329-332.

Foegeding, P. M. \& FulP, M. L. (1988). Comparison of coats and surface-dependent properties of Bacillus cereus $\mathrm{T}$ prepared in two sporulation environments. Journal of Applied Bacteriology 65, 249-259.

Hachisuka, Y., KozUKa, S. \& TsujikaWa, M. (1984). Exosporia and appendages of spores of Bacillus species. Microbiology and Immunology 28, 619-624.

Hitchins, A. D., GReENe, R. A. \& SLePeCKy, R. A. (1972). Effect of carbon source on size and associated properties of Bacillus megaterium spores. Journal of Bacteriology 110, 392-401.

Koshikawa, T., Beaman, T. C., Pankratz, H. S., Nakashio, S., Corner, T. R. \& GerhardT, P. (1984). Resistance, germination, and permeability correlates of Bacillus megaterium spores successively divested of integument layers. Journal of Bacteriology 159, 624-632.

KozUka, S. \& Tochikubo, K. (1985). Properties and origin of filamentous appendages on spores of Bacillus cereus. Microbiology and Immunology 29, 21-37.

Kutima, P. M. \& Foegeding, P. M. (1987). Involvement of the spore coat in germination of Bacillus cereus $\mathrm{T}$ spores. Applied and Environmental Microbiology 53, 47-52.

LINDAHL, M., FARIS, A., WADSTRÖM, T. \& HJERTÉN, S. (1981). A new test based on 'salting out' to measure relative surface hydrophobicity of bacterial cells. Biochimica et biophysica acta 667, 471-476.
Matz, L. L., Beaman, T. C. \& Gerhardt, P. (1970) Chemical composition of exosporium from spores of Bacillus cereus. Journal of Bacteriology 101, 196-201.

OHEY, D. F. \& MURRELL, W. G. (1962). Formation and structure of the spore of Bacillus coagulans. Journal of Cell Biology 14, 111-123.

Rosenberg, E., Brown, D. R. \& Demain, A. L. (1985). The influence of gramicidin $S$ on hydrophobicity of germinating Bacillus brevis spores. Archives of Microbiology 142, 51-54.

Rosenberg, M., Gutnick, D. \& Rosenberg, E. (1980). Adherence of bacteria to hydrocarbons: a simple method for measuring cell-surface hydrophobicity. FEMS Microbiology Letters 9, 29-33.

Setlow, P. \& KornberG, A. (1969). Biochemical studies of bacterial sporulation and germination. XVII. Sulfhydryl and disulfide levels in dormancy and germination. Journal of Bacteriology 100, 11551160 .

SMYTH, C. J., JonsSon, P., Olsson, E., SÖDERLind, O., Rosengren, J., HJertén, S. \& WadSTRÖM, T. (1978). Differences in hydrophobic surface characteristics of porcine enteropathogenic Escherichia coli with or without $\mathrm{K} 88$ antigen as revealed by hydrophobic interaction chromatography. Infection and Immunity 22, 462-472.

Sousa, J. C. F., Silva, M. T. \& Balassa, G. (1976). An exosporium-like outer layer in Bacillus subtilis spores. Nature, London 263, 53-54.

TaKumi, K., Kinouchi, T. \& KaWaTA, T. (1979). Isolation and partial characterization of exosporium from spores of a highly sporogenic mutant of Clostridium botulinum type A. Microbiology and Immunology 28, 443-454.

Waites, W. M., Stansfield, R. \& Bayliss, C. E. (1979). The effect of sporulation medium on the structure and heat resistance of spores of Clostridium bifermentans. FEMS Microbiology Letters 5, 365-368.

WARTH, A. D., OHEY, D. F. \& MURRELL, W. G. (1963). The composition and structure of bacterial spores. Journal of Cell Biology 16, 579-592. 